Western University

Scholarship@Western

$1-1-2020$

\title{
Using fMRI to investigate the potential cause of inverse oxygenation reported in fNIRS studies of motor imagery
}

\author{
Androu Abdalmalak \\ Lawson Health Research Institute \\ Daniel Milej \\ Lawson Health Research Institute \\ David J. Cohen \\ Western University \\ Udunna Anazodo \\ Lawson Health Research Institute \\ Tracy Ssali \\ Lawson Health Research Institute
}

See next page for additional authors

Follow this and additional works at: https://ir.lib.uwo.ca/brainscanpub

Part of the Neuroscience and Neurobiology Commons

\section{Citation of this paper:}

Abdalmalak, Androu; Milej, Daniel; Cohen, David J.; Anazodo, Udunna; Ssali, Tracy; Diop, Mamadou; Owen, Adrian M.; and St. Lawrence, Keith, "Using fMRI to investigate the potential cause of inverse oxygenation reported in fNIRS studies of motor imagery" (2020). BrainsCAN Publications. 9.

https://ir.lib.uwo.ca/brainscanpub/9 


\section{Authors}

Androu Abdalmalak, Daniel Milej, David J. Cohen, Udunna Anazodo, Tracy Ssali, Mamadou Diop, Adrian M. Owen, and Keith St. Lawrence 
Research article

\title{
Using fMRI to investigate the potential cause of inverse oxygenation reported in fNIRS studies of motor imagery
}

\author{
Androu Abdalmalak ${ }^{\mathrm{a}, \mathrm{b}, *}$, Daniel Milej ${ }^{\mathrm{a}, \mathrm{b}}$, David J. Cohen ${ }^{\mathrm{b}}$, Udunna Anazodo ${ }^{\mathrm{a}, \mathrm{b}}$, Tracy Ssali ${ }^{\mathrm{a}, \mathrm{b}}$, \\ Mamadou Diop ${ }^{\mathrm{a}, \mathrm{b}}$, Adrian M. Owen ${ }^{\mathrm{c}}$, Keith St. Lawrence ${ }^{\mathrm{a}, \mathrm{b}}$ \\ ${ }^{a}$ Imaging Program, Lawson Health Research Institute, London, Ontario, N6A 4V2, Canada \\ ${ }^{\mathrm{b}}$ Department of Medical Biophysics, Western University, London, Ontario, N6A 5C1 Canada \\ ${ }^{\mathrm{c}}$ The Brain and Mind Institute, Western University, London, Ontario, N6A 5B7, Canada
}

\section{A R T I C L E I N F O}

\section{Keywords:}

Functional near-infrared spectroscopy

Motor imagery

Inverse oxygenation

Functional magnetic resonance imaging

Brain-Computer interface

\begin{abstract}
A B S T R A C T
Motor imagery (MI) is a commonly used cognitive task in brain-computer interface (BCI) applications because it produces reliable activity in motor-planning regions. However, a number of functional near-infrared spectroscopy (fNIRS) studies have reported the unexpected finding of inverse oxygenation: increased deoxyhemoglobin and decreased oxyhemoglobin during task periods. This finding questions the reliability of fNIRS for BCI applications given that MI activation should result in a focal increase in blood oxygenation. In an attempt to elucidate this phenomenon, fMRI and fNIRS data were acquired on 15 healthy participants performing a MI task. The fMRI data provided global coverage of brain activity, thus allowing visualization of all potential brain regions activated and deactivated during task periods. Indeed, fMRI results from seven subjects included activation in the primary motor cortex and/or the pre-supplementary motor area during the rest periods in addition to the expected activation in the supplementary motor and premotor areas. Of these seven subjects, two showed inverse oxygenation with fNIRS. The proximity of the regions showing inverse oxygenation to the motor planning regions suggests that inverse activation detected by fNIRS may likely be a consequence of partial volume errors due to the sensitivity of the optodes to both primary motor and motor planning regions.
\end{abstract}

\section{Introduction}

Brain-computer interfaces (BCIs) are devices that can be used to bridge the gap between thoughts and actions, allowing patients with physical impairment to control external devices or communicate with the outside world [1]. BCI applications often use motor imagery (MI), which relies on participants actively imagining movement and simultaneously recording brain activity from the motor-planning regions of the brain (i.e. the supplementary motor area (SMA) and premotor cortex (PMC)) $[2,3]$. There has been a growing interest in using functional near-infrared spectroscopy (fNIRS) for BCI applications since the technology is portable, safe, and can detect cortical brain activity associated with MI $[4-5,6,7]$. Analogous to functional magnetic resonance imaging (fMRI) based on blood oxygen level dependent (BOLD) contrast, fNIRS maps regional brain activity by detecting activation-induced changes in oxyhemoglobin and deoxyhemoglobin concentrations $[8-9,10]$. The resulting hemodynamic response (i.e., increased oxyhemoglobin and decreased deoxyhemoglobin) is a result of regional increases in blood flow and blood volume that exceed the corresponding increase in regional metabolic demand [11]. These changes in the concentration of oxyhemoglobin and deoxyhemoglobin can be determined by measuring absorption changes at two or more wavelengths of light [12]. A number of studies involving both healthy and patient populations have reported the feasibility of fNIRS for BCI applications [1,13-14,15].

Although promising, questions regarding inter-subject variability need to be addressed if fNIRS is to become a reliable BCI. One concern is the phenomenon of 'inverse oxygenation' observed during MI tasks in a number of previous studies [16,17]. Inverse oxygenation is the reverse of the expected hemoglobin signal changes during a task; namely, the concentration of oxyhemoglobin decreases while the concentration of deoxyhemoglobin increases. Holper and colleagues reported this phenomenon in up to $50 \%$ of participants, which presents a potential challenge in designing a generic BCI based on the conventional hemodynamic response during a task. This unexplained finding has been attributed to the complexity of MI task; i.e. inverse oxygenation was observed more frequently during simple versus complex MI tasks [16]. Kempny et al. also reported similar changes in the signal during motor

\footnotetext{
* Corresponding author at: Imaging Program, Lawson Health Research Institute, London, Ontario, N6A 4V2, Canada.

E-mail address: aabdalma@uwo.ca (A. Abdalmalak).
} 
imagery in some patients with disorders of consciousness [17]. A second concern is the lack of consensus regarding the best location for optodes to detect MI activity, with numerous studies placing a grid of optodes over the entire motor cortex $[17,18]$ despite fMRI studies showing that the secondary motor regions are the most consistently activated $[3,19,20]$.

To this end, the goals of this study were to investigate the prevalence of inverse oxygenation during MI and to provide a plausible explanation by comparing fNIRS results to whole-brain fMRI results. It was our hypothesis that inverse oxygenation could be caused by inadvertent movement during rest periods, leading to activation in the primary motor cortex. Considering this region is adjacent to the motor planning regions, this out-of-phase activation could be misinterpreted as inverse oxygenation due to the poor spatial resolution of fNIRS. That is, probes that are sensitive to activation in both areas are prone to partial volume errors, leading to suboptimal recording of MI activity. To test this hypothesis, fNIRS and fMRI were acquired on a cohort of fifteen healthy participants performing a well-established MI task $[19,21]$. Having access to fMRI data allowed for the visualization of potential changes in brain activity during both task and rest periods. To further test our hypothesis, Monte Carlo simulations were conducted on a layered head model with the primary and secondary motor areas defined in order to assess the relative probe sensitivity to each region at various probe locations. This allowed us to investigate if incorrect probe placement could lead to significant signal contributions (i.e. contamination) from the primary motor cortex.

The data presented in this study were acquired as part of a previous study conducted to assess the sensitivity and feasibility of fNIRS to detect brain activity during MI [22]. The purpose of the previous study was to investigate the potential advantage of time-resolved (TR) detection for enhancing the sensitivity of fNIRS to MI-related brain activation. Using fMRI as the standard, it was shown that TR-fNIRS increased detection sensitivity from $64 \%$ to $93 \%$. At the time of that publication, the occurrence of inverse oxygenation had not been investigated since the fMRI data were only analyzed to look for the contrast task greater than rest. Possible activation in brain regions during the rest periods (i.e., rest $>$ task) was not investigated.

\section{Materials and methods}

Fifteen healthy subjects were recruited ( 5 females and 10 males, 22-34 years, mean age $=26$ ), with each subject undergoing sequential fMRI and fNIRS scans involving a MI session of imagining playing tennis. The protocol consisted of a $30 \mathrm{~s}$ baseline period followed by 5 cycles of $30 \mathrm{~s}$ alternating blocks of rest and MI, for a total experimental time of 5:30 min [19]. The participants were instructed to imagine playing a vigorous game of tennis every time they heard the word "tennis" and to relax when they heard the word "rest". They were also instructed to keep their eyes closed and stay as still as possible throughout the entire study. This study was approved by the Research Ethics Board at Western University.

\subsection{Data acquisition}

The fMRI scans were done on a 3 Tesla Biograph mMR scanner (Siemens Healthcare, Erlangen, Germany) at St. Joseph's Health Care Centre, London, Ontario using a 32-channel receive-only head coil. Magnetization prepared rapid gradient echo (MPRAGE) images (echo time $=2.98 \mathrm{~ms}$, recovery time $=2000 \mathrm{~ms}, \quad \mathrm{FA}=9^{\circ}, \quad$ voxel size $=1 \times 1 \times 1 \mathrm{~mm}$ ) were first acquired, followed by acquiring functional data using an echo-planar imaging (EPI) sequence (echo time $=30 \mathrm{~ms}$, recovery time $=3000 \mathrm{~ms}, \quad \mathrm{FA}=90^{\circ}$, slice thickness $=3 \mathrm{~mm}$, voxel size $=3 \times 3 \times 3 \mathrm{~mm}$, total number of scans $=110$ ). An MRI-compatible headset was used to deliver the verbal cues to the participants.

The fNIRS system was an in-house built time-resolved system with four detection channels that operated at 760 and $830 \mathrm{~nm}[23,24]$. To interrogate the SMA and PMC, the fNIRS emission and detection channels were centered over $\mathrm{FCz}$ according to the international system for EEG electrode placement. The fibers were secured to the head using a 3D printed holder and an EEG cap (EasyCap) [25]. Throughout each MI experiment, distributions of times-of-flight of photons (DTOFs) were continuously recorded every $300 \mathrm{~ms}$ with a temporal resolution of $16 \mathrm{ps}$.

\subsection{Data analysis}

Functional MRI data were analyzed using SPM8 (Wellcome Trust Center for Neuroimaging, University College London, UK). The functional images were realigned, spatially normalized to the EPI template, smoothed and filtered to correct for any baseline drifts. Single subject analysis was performed with the condition of each scan defined as belonging to rest or MI. Whole brain analysis was performed to determine statistically significant brain activity, with the statistical threshold set to a false discovery rate (FDR) corrected $p<0.05$. The contrasts of task $>$ rest and rest $>$ task were generated to investigate which brain regions were activated during MI and during rest periods, respectively. Finally, for participants who failed to show activity at the whole brain level for the contrast of task > rest, small volume correction was used with spheres set over the SMA and PMC [20].

The fNIRS data were analyzed using code developed in MATLAB. To begin with, the integration limits were set to $10 \%$ and $1 \%$ of the arrival time corresponding to the peak of the DTOF [26], and the first three statistical moments of each DTOF were calculated since higher moments provide greater sensitivity to late-arriving photons [27]. Subsequent analysis was conducted using the first moment (the mean timeof-flight, $\langle t\rangle$ ) as it was previously shown to provide the best balance between depth enhancement and detecting MI-related brain activation [22]. The $\langle t\rangle$ time courses for each channel were independently preprocessed using functions adapted from the SPM-fNIRS toolbox. Briefly, all $\langle t\rangle$ time courses were corrected for motion artifacts using the movement artifact reduction algorithm (MARA) approach [28]. Next, the signals were filtered using a bandstop filter with stopband frequencies between 0.08 and $1.5 \mathrm{~Hz}$, detrended to remove any slow drifts in the signals, converted to hemoglobin signals using sensitivity factors [24] and analyzed using the General Linear Model [22]. Each timecourse was visually inspected after each pre-processing step to ensure the quality of the signals were adequate for further processing. Inverse oxygenation was defined as a significant $(p<0.05)$ increase in deoxyhemoglobin and a concurrent decrease in oxyhemoglobin during the task periods.

\subsection{Monte Carlo simulations}

Simulations were conducted in Matlab using a mesh-based Monte Carlo method [29]. A 5-layer segmented adult head model [30] available from the NIRFAST website was used with optical properties previously reported by Jäger and colleagues [31]. Two regions of interests were defined and segmented using 3D slicer: the secondary motor regions, consisting of the SMA and PMC, and the primary motor region (Fig. 1). The initial positions of the emission and detection fibers were the same as those used in the MI activation studies (i.e., the emission fiber centered over FCz). Next, the fibers were moved posterior to $\mathrm{FCz}$ in $0.5 \mathrm{~cm}$ increments until the emission fiber was $2 \mathrm{~cm}$ from the correct position. This was done to simulate incorrect positioning of the probes relative to secondary motor regions. For each position, 100 million photons were injected as a delta function using a single point source at the source location and diffusively reflected photons were recorded at each of the four detector locations with a detector diameter of $3 \mathrm{~mm}$. Contamination from the primary motor cortex (i.e. sensitivity to the primary motor cortex) was calculated as the ratio of the sum of photon pathlengths that interrogated the primary motor cortex to the sum of 


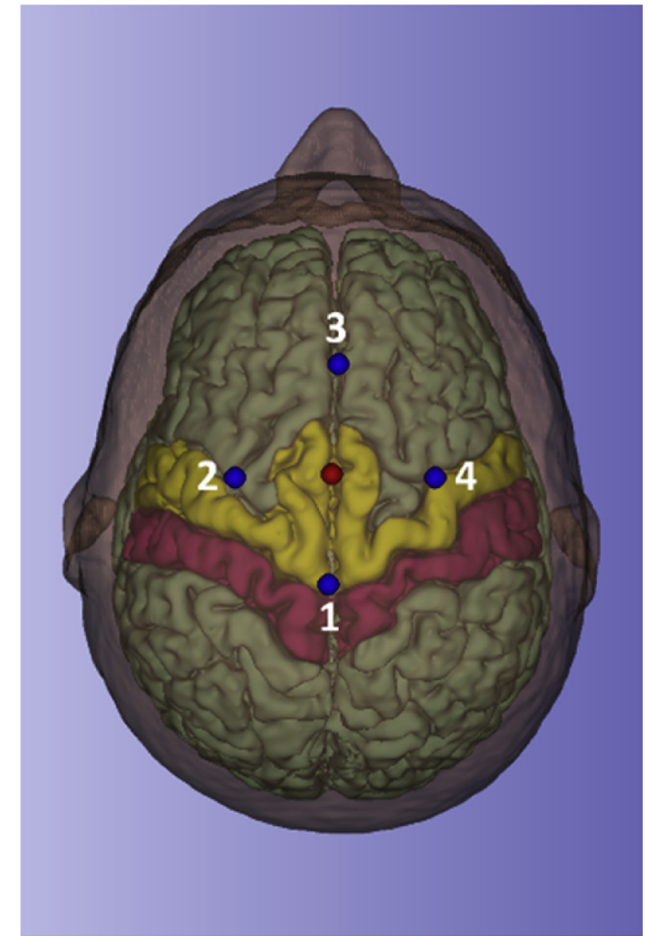

Fig. 1. Segmented head model with the simulated positions of the optodes shown: red for the emission fiber (placed over FCz) and the blue for the four detection fibers, which were placed in a cross orientation (detector 1 (back), detector 2 (left), detector 3 (front), detector 4 (right)) at a source-detector distance of $3 \mathrm{~cm}$. The SMA and PMC are also shown in yellow and the primary motor cortex in magenta.

pathlengths that interrogated secondary and primary motor areas.

\section{Results}

\section{1. fNIRS and fMRI}

Expected activation in the SMA and/or PMC was detected in 13 of the 15 participants by both fMRI and fNIRS, as reported previously [22]. Whole-brain analysis of the fMRI data revealed that during the rest periods (i.e., rest $>$ task), seven subjects (two females) had activity in the primary motor cortex, six subjects had activity in the visual cortex, and three showed activity in the pre-SMA (Fig. 2). This figure also shows the fNIRS channels with significant increases and decreases in oxyhemoglobin (red and blue dots, respectively).

Of these seven subjects, only two (subjects 1 and 4) showed inverse oxygenation with fNIRS (one female and one male). The hemoglobin time courses for these two subjects averaged across all 5 trials are shown in Fig. 3, along with the time courses from another two subjects who showed the expected oxygenation patterns. For subject 1 (first row), three channels showed inverse oxygenation (channels $1,2 \& 4$ ), while for subject 4 (second row), two channels showed inverse oxygenation (channels 1 \& 3). Fig. 4 presents the average oxy- and deoxyhemoglobin time courses across all subjects who had the expected oxygenation response and for the two participants who had inverse oxygenation. Note, the inverse oxygenation time courses were generated from channels that had a significant decrease in oxygenation.

\subsection{Monte Carlo simulations}

The sensitivity of each detector to the primary motor cortex for each of the five locations, starting at $\mathrm{FCz}$ and moving posteriorly by $0.5 \mathrm{~cm}$, is presented in Table 1. As expected, detector 1 placed at the back showed the highest sensitivity to the primary motor cortex for each position.

\section{Discussion}

The goals of this study were to investigate the prevalence of inverse oxygenation during MI and to provide a plausible explanation for this phenomenon. By acquiring fMRI data for each participant, we were able to provide global coverage of brain activity to assess which regions were activated during task periods, as well as possible activation during rest periods. Considering the BOLD contrast is tightly coupled to synaptic activity, positive contrast reflects the expected increase in activity during task periods, while negative contrast reflects higher activity during rest periods [32]. An example of the latter is the negative BOLD signal commonly observed in regions of the default mode network during cognitive tasks [33]. As previously reported, increased brain activity in the secondary motor regions during motor imagery was observed in 13 of 15 participants by both fMRI and fNIRS based on the analysis of $\langle t\rangle$ data [22,25]. Reanalyzing the fMRI data in terms of rest signal greater than task signal, and using FDR-corrected threshold, revealed activation in the primary motor cortex during the rest periods for seven participants. Similarly, inverse oxygenation was found by fNIRS in two of these subjects. To demonstrate that the fMRI deactivation findings were not dependent on the statistical tests used, the analysis was repeated using corrected threshold Gaussian random field. Significant activation remained for 6 of the 7 subjects shown in Fig. 2. Deactivation results for subject 7 failed this more conservation threshold, which is not unexpected considering activation based on FDR was considerably weaker compared to the other subjects.

The relationship between regional brain activity and BOLD contrast provides a plausible explanation for the inverted fNIRS signals (i.e. increased deoxyhemoglobin concentration) observed in this study [16]. The fMRI results showed the expected positive contrast in motor planning regions during the MI task, while negative contrast (i.e. higher during the rest periods) was found predominately in the primary motor cortex and the visual cortex in seven of fifteen subjects. These inverted signals were likely due to inadvertent motion and eye opening during the rest periods, possibly as a de-stressing mechanism after performing MI. Considering that the fNIRS signals should mirror the BOLD contrast, the fMRI results suggest that inverse oxygenation observed by fNIRS in two subjects was likely due to increased activity during the rest periods and was not related to the MI task. This inadvertent activity during the rest periods is compounded by partial volume errors due to the poorer spatial resolution of fNIRS and uncertainties associated with probe placement on the scalp. That is, the relative contributions of MI-related activation in the motor planning regions and the out-of-phase activation in the primary motor cortex activity will vary depending on the sensitivity of the probes to these adjacent brain regions and on the relative strength of the activation in these regions. Uncertainties with respect to probe placements have been reported in the literature and are attributed to human error and anatomical variability between subjects [16] when relying on a 10-20 template to define probe locations. That being said, the fact that inverse oxygenation was only detected in two subjects by fNIRS suggests the probes were generally positioned properly, such that they had greater sensitivity to secondary motor regions than the primary motor cortex.

The results from the Monte Carlo simulations confirm the hypothesis that incorrectly placing the probes can lead to partial volume errors. That is, there can be significant signal contributions from the primary motor cortex due to its close proximity to motor planning regions and the relatively large source-detector separation used in the current study $(3 \mathrm{~cm})$. As expected, the most posterior probe (detector 1 ) was the most sensitive to the primary motor cortex, with more than half the signal coming from this region if the probe position was $2 \mathrm{~cm}$ posterior to FCz. Previous work has shown that anatomical variations between subjects can lead to localization errors of up to $18 \mathrm{~mm}$ [34]. In 
a)
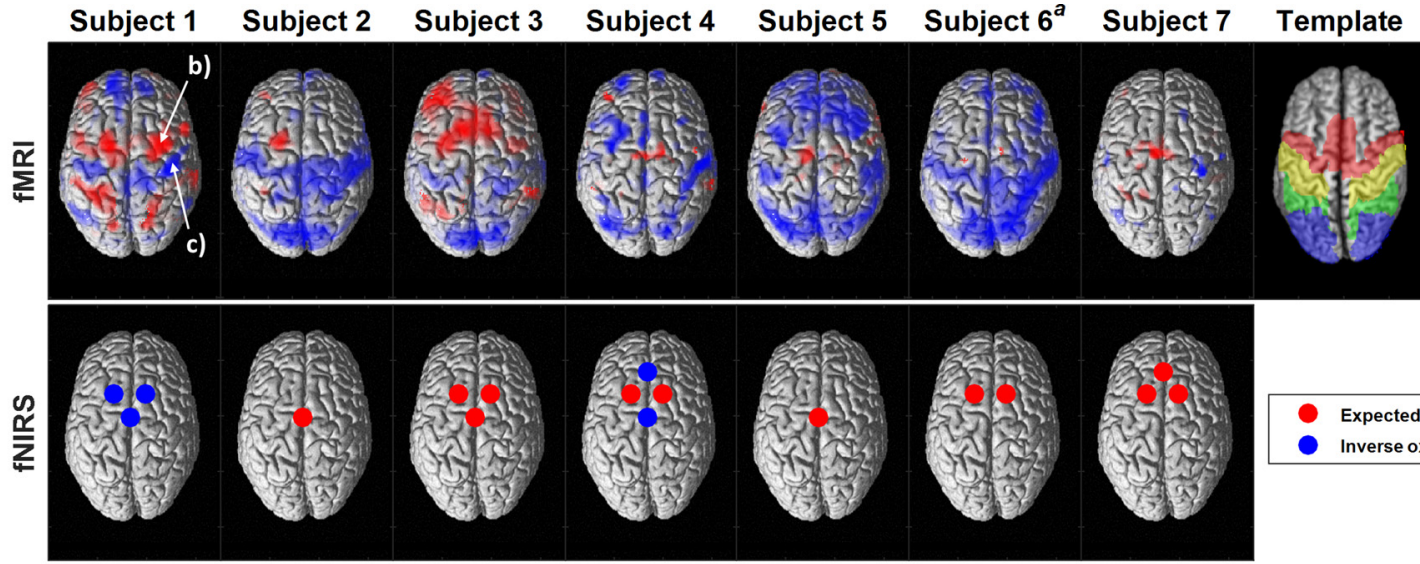

Expected oxygenation

Inverse oxygenation

b)

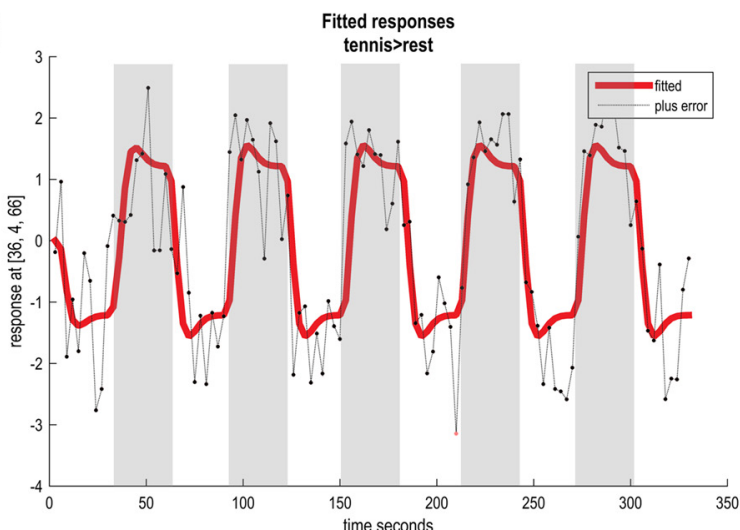

c)

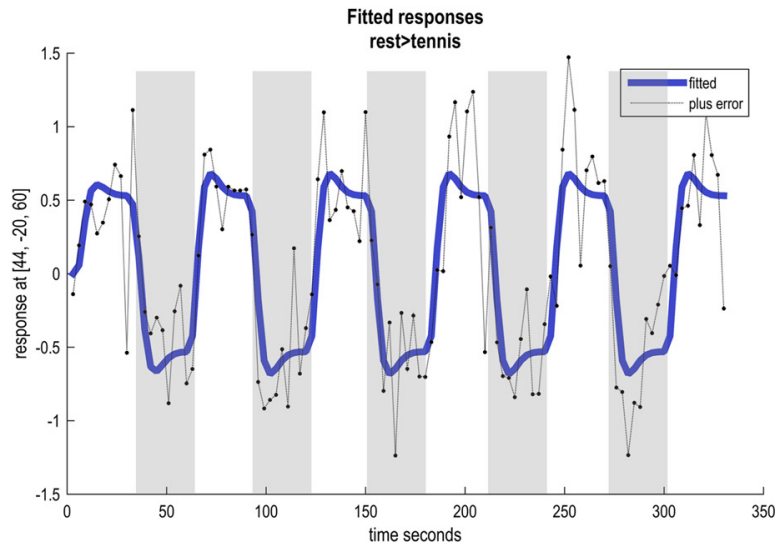

Fig. 2. (a) fMRI and fNIRS activity for 7 subjects overlaid on a single-subject T1 template. For the fMRI results, the brain regions activated during the task period

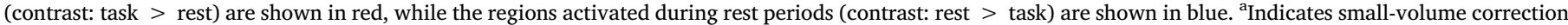
for the contrast of task > rest. A template of the brain regions activated across these subjects is provided with the brain regions colour-coded as follows: red PreSMA, SMA and PMC; yellow primary motor cortex; green parietal cortex; and blue visual cortex. This template is provided to aid in understanding the fMRI results. For the fNIRS results, red dots indicate channels that showed significant typical or expected oxygenation changes while blue dots indicate channels that showed significant inverse oxygenation. fMRI time courses for subject 1 obtained from two different voxels are also shown with (b) showing the predicted responses during the task and (c) showing the predicted inverse oxygenation (contrast: rest $>$ task). The grey boxes indicate the task periods.
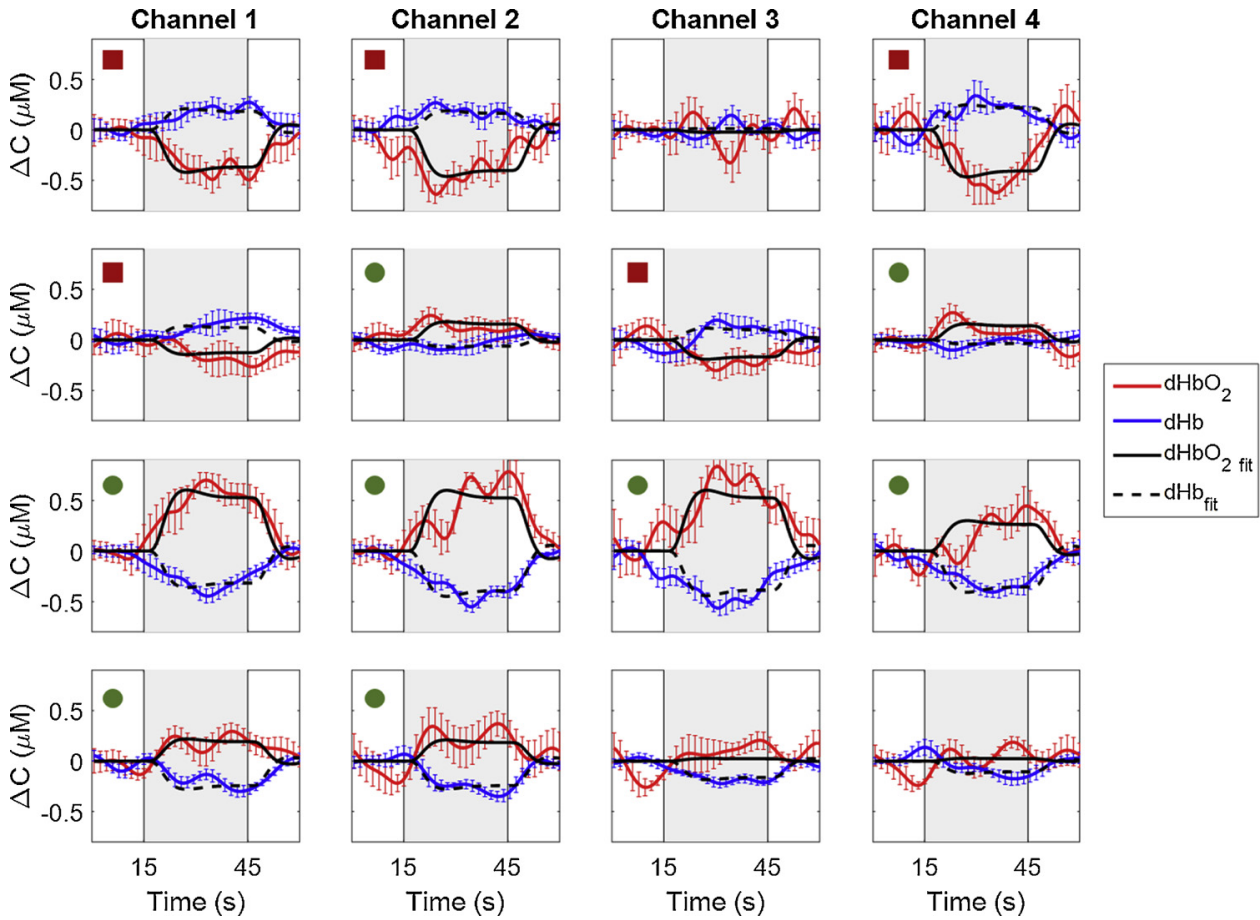

Fig. 3. Changes in the concentration of oxy(red) and deoxyhemoglobin (blue) for each channel averaged across 5 trials. The model fit for oxy- and deoxyhemoglobin are represented by the solid and dashed lines, respectively. Each row represents data from one subject. Red squares indicate channels that had a significant decrease in oxy- and a concurrent increase in deoxy-hemoglobin. Conversely, green circles indicate channels that showed the expected significant increase in oxy- and concurrent decrease in deoxy-hemoglobin. The error bars represent the standard error of the mean across trials and the grey boxes indicate the task period. 


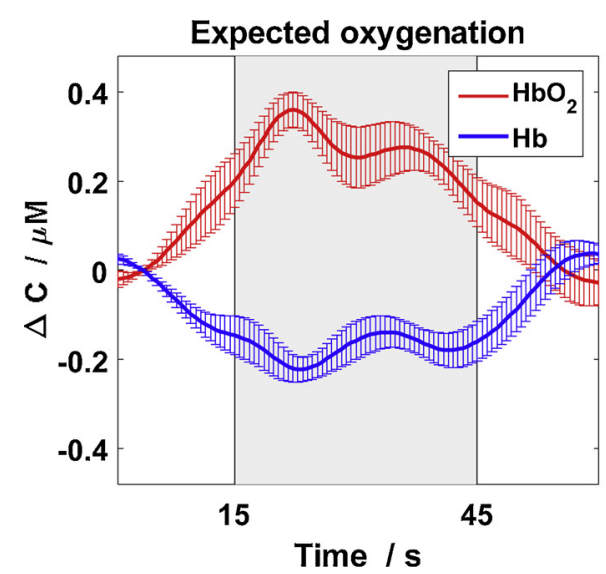

Time / s

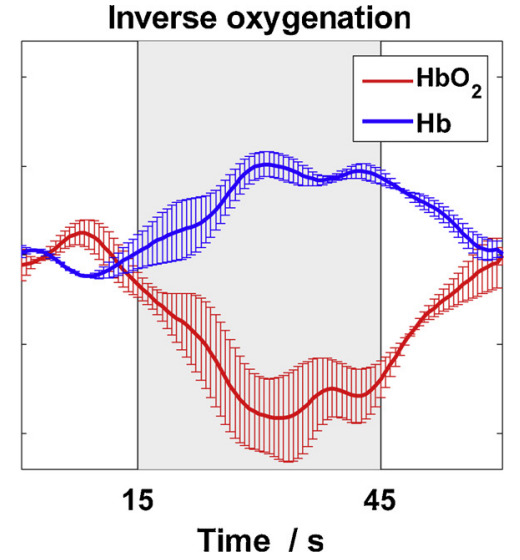

Fig. 4. Changes in the concentrations of oxyhemoglobin (red) and deoxyhemoglobin (blue) averaged across all five cycles and all channels. The data were divided into the expected oxygenation response or the inverted oxygenation response found in two subjects. The error bars represent the standard error of mean across all participants in the two groups ( $\mathrm{n}=13$ for the expected activation and $\mathrm{n}=2$ for inverse oxygenation). Grey boxes indicate the task periods.
Table 1

Monte Carlo predictions of the sensitivity of each channel to the primary motor cortex relative to the secondary motor areas.

\begin{tabular}{lllll}
\hline Position & \multicolumn{3}{l}{ Sensitivity to the primary moyor cortex (\%) } \\
\cline { 2 - 5 } & $\begin{array}{l}\text { Detector } 1 \\
\text { (back) }\end{array}$ & $\begin{array}{l}\text { Detector } 2 \\
\text { (left) }\end{array}$ & $\begin{array}{l}\text { Detector } 3 \\
\text { (front) }\end{array}$ & $\begin{array}{l}\text { Detector } 4 \\
\text { (right) }\end{array}$ \\
\hline $\mathrm{FCz}$ & 17.8 & 5.1 & 1.7 & 6.4 \\
$\mathrm{FCz}+0.5 \mathrm{~cm}$ & 24.4 & 7.1 & 2.6 & 10.1 \\
$\mathrm{FCz}+1.0 \mathrm{~cm}$ & 32.6 & 12.0 & 3.3 & 13.8 \\
$\mathrm{FCz}+1.5 \mathrm{~cm}$ & 39.2 & 17.9 & 4.7 & 19.0 \\
$\mathrm{FCz}+2 \mathrm{~cm}$ & 51.4 & 27.1 & 7.8 & 31.5 \\
\hline
\end{tabular}

addition, relying on the 10-20 template has been shown to lead to errors of up to $13 \mathrm{~mm}$ from the true 10-20 landmark [35]. Furthermore, spatial variability of the 10-20 system with race has been reported by Noh et al. with key anatomical locations being significantly different between Asian and Caucasian decedents [36]. However, this difference likely did not contribute to the inverse oxygenation reported in the current study as both subjects were Caucasian.

Contamination will also depend on if there is inadvertent movement during rest periods and its relative signal strength compared to MI activity in the secondary motor regions. As a result, it should be emphasized that although more than $50 \%$ of the signal could originate from the primary motor at larger positional errors, the primary motor cortex must be activated during the rest periods in order to detect inverse oxygenation. Consistent with the predictions of the Monte Carlo results, inverse oxygenation detected for two participants was found for detector 1 , which was the probe location closest to the primary motor cortex. Inverse oxygenation was also found for subject 1 for the two lateral channels, and the simulations revealed that a substantial fraction of the signal for these channels could come from the primary motor cortex. Interestingly, channel 3, the most distal channel, also had significant inverse oxygenation for subject 4 . The fMRI maps for this subject revealed activity during the rest periods in the pre-SMA a brain region involved in higher-level planning such as switching actions and selective inhibition [37,38], suggesting conscious inhibition of the motor imagery task during the rest periods. To further confirm the Monte Carlo predictions, activation data were acquired from three subjects for a sensory task (brushing the palm) that activates the somatosensory cortex, but not motor-planning regions [39]. Activation was not detected when the probes were placed over FCz (data not shown). In contrast, when the probes were placed $2 \mathrm{~cm}$ posterior, as per the Monte Carlo simulations, activation was detected for all participants from channel 2, which interrogated the ipsilateral somatosensory cortex.

Functional NIRS-BCI studies using various MI paradigms have focused on placing probes covering the entire motor cortex $[6,18]$. For instance, Kempny et al. placed their probes over C3 and C4 to get MI activity in disorder of consciousness (DoC) patients, and they reported inverse oxygenation in around $40 \%$ of patients [17]. Similarly, Holper et al. reported inverse oxygenation in up to $50 \%$ of healthy participants performing a MI task [16]. Although we cannot conclude that inverse activity reported in those studies was due to subject motion, the percentage of subjects with inverse oxygenation is similar to the percentage of participants in the current study who showed activation during rest periods in the primary motor cortex with fMRI. These results suggest that it is critical to focus on probes placed over the secondary motor regions to reduce signal contamination from the primary motor cortex. In the current study, the probes were centered over $\mathrm{FCz}$ to optimize the sensitivity to functional activation in the SMA and PMC.

A limitation with this study is that both imaging sessions were not performed simultaneously and hence it is not possible to conclude that participants were moving to the same extent during both sessions. Another potential limitation is the poor spatial resolution of the fourchannel TR systems. While a higher density of optodes could enhance the spatial resolution and aid in resolving activation in adjacent brain regions $[37,38]$, previous work has shown that the four-channel approach is well suited for MI tasks given the highly localized activation (i.e. SMA and PMC) and the enhanced depth sensitivity provided by TR detection [22]. The fact that only two participants showed inverse oxygenation with TR-fNIRS supports the argument that the fourchannel approach is well suited for detecting MI activation. Finally, the small sample size of subjects that showed inverse oxygenation with TRfNIRS (i.e. 2 subjects) could be conceived as a potential limitation. However, it should be emphasized that the main conclusions of this study are drawn from the fMRI results, where seven participants showed activation in the primary motor cortex during the rest periods. As a result, the effective sample size is nearly $50 \%$ of all the subjects recruited (7/15 participants).

In conclusion, this study demonstrated that a likely cause of inverse oxygenation detected by fNIRS during MI is due to inadvertent subject movement during rest periods. To avoid this confounding effect, it is important to place the probes over the motor planning regions instead of the primary motor cortex.

\section{Funding}

This research was funded by the Canada Excellence Research Chairs (CERC) program, Natural Science and Engineering Research Council (NSERC) of Canada and Canadian Institutes of Health Research (CIHR).

\section{Declaration of Competing Interest}

The authors declare no conflict of interest. 


\section{Acknowledgment}

The authors would like to thank John Butler and Heather Biernaski for their assistance with running the MRI scanner.

\section{References}

[1] N. Naseer, K.-S. Hong, fNIRS-based brain-computer interfaces : a review, Front Hum. Neurosci. 9 (2015) 1-15, https://doi.org/10.3389/fnhum.2015.00003.

[2] L. Naci, M.M. Monti, D. Cruse, A. Kübler, B. Sorger, R. Goebel, B. Kotchoubey, A.M. Owen, Brain-computer interfaces for communication with nonresponsive patients, Ann. Neurol. 72 (2012) 312-323, https://doi.org/10.1002/ana.23656.

[3] A.M. Owen, M.R. Coleman, M. Boly, M.H. Davis, S. Laureys, J.D. Pickard, Detecting Awareness in the Vegetative State, Science (80-) 313 (2006) 1402 abstract http:// science.sciencemag.org/content/313/5792/1402.

[4] R. Sitaram, H. Zhang, C. Guan, M. Thulasidas, Y. Hoshi, A. Ishikawa, K. Shimizu, N. Birbaumer, Temporal classification of multichannel near-infrared spectroscopy signals of motor imagery for developing a brain-computer interface, Neuroimage 34 (2007) 1416-1427, https://doi.org/10.1016/j.neuroimage.2006.11.005.

[5] A.M. Batula, J.A. Mark, Y.E. Kim, H. Ayaz, Comparison of brain activation during motor imagery and motor movement using fNIRS, Comput. Intell. Neurosci. 2017 (2017), https://doi.org/10.1155/2017/5491296.

[6] S.M. Coyle, T.E. Ward, C.M. Markham, Brain-computer interface using a simplified functional near-infrared spectroscopy system, J. Neural Eng. 4 (2007) 219-226, https://doi.org/10.1088/1741-2560/4/3/007.

[7] M. Rupawala, H. Dehghani, S.J.E. Lucas, P. Tino, D. Cruse, Shining a light on awareness: a review of functional near-infrared spectroscopy for prolonged disorders of consciousness, Front. Neurol. 9 (2018) 350, https://doi.org/10.3389/ fneur.2018.00350.

[8] G. Strangman, J.P. Culver, J.H. Thompson, D.A. Boas, A quantitative comparison of simultaneous BOLD fMRI and NIRS recordings during functional brain activation, Neuroimage 17 (2002) 719-731 http://www.ncbi.nlm.nih.gov/entrez/query.fcgi? $\mathrm{cmd}=$ Retrieve\%7B\&\%7Ddb = PubMed\%7B\&\%7Ddopt $=$ Citation\%7B\&\%7Dlist $\% 7 B$ \% $\% 7$ Duids $=12377147$

[9] F. Moreau, R. Yang, V. Nambiar, A.M. Demchuk, J.F. Dunn, Near-infrared measurements of brain oxygenation in stroke, Neurophotonics 3 (2016) 031403, , https://doi.org/10.1117/1.NPh.3.3.031403.

[10] H.N. Modi, H. Singh, F. Orihuela-Espina, T. Athanasiou, F. Fiorentino, G.-Z. Yang, A. Darzi, D.R. Leff, Temporal Stress in the Operating Room: Brain Engagement Promotes "Coping" and Disengagement Prompts "Choking", Ann. Surg. 267 (2018) 683-691 https://journals.lww.com/annalsofsurgery/Fulltext/2018/04000/ Temporal Stress in the Operating Room Brain.14.aspx.

[11] G.H. Glover, Overview of functional magnetic resonance imaging, Neurosurg. Clin. N. Am. 22 (2011) 133-139, https://doi.org/10.1016/j.nec.2010.11.001.

[12] D.A. Boas, C.E. Elwell, M. Ferrari, G. Taga, Twenty years of functional near-infrared spectroscopy: introduction for the special issue, Neuroimage. 85 (2014) 1-5, https://doi.org/10.1016/j.neuroimage.2013.11.033.

[13] U. Chaudhary, B. Xia, S. Silvoni, L.G. Cohen, N. Birbaumer, Brain-Computer Interface-Based Communication in the Completely Locked-In State, PLoS Biol. 15 (2017) 1-25, https://doi.org/10.1371/journal.pbio.1002593.

[14] G. Gallegos-Ayala, A. Furdea, K. Takano, C.A. Ruf, H. Flor, N. Birbaumer, Brain communication in a completely locked-in patient using bedside near-infrared spectroscopy, Neurology 82 (2014) 1930-1932, https://doi.org/10.1212/WNL. 0000000000000449 .

[15] A. Abdalmalak, D. Milej, L. Norton, D.B. Debicki, T. Gofton, M. Diop, A.M. Owen, K.St. Lawrence, Single-session communication with a locked-in patient by functional near-infrared spectroscopy, Neurophotonics 4 (2017) 1, https://doi.org/10. 1117/1.NPh.4.4.040501.

[16] L. Holper, D.E. Shalóm, M. Wolf, M. Sigman, Understanding inverse oxygenation responses during motor imagery: a functional near-infrared spectroscopy study, Eur. J. Neurosci. 33 (2011) 2318-2328, https://doi.org/10.1111/j.1460-9568. 2011.07720.x.

[17] A.M. Kempny, L. James, K. Yelden, S. Duport, S. Farmer, E.D. Playford, A.P. Leff, Functional near infrared spectroscopy as a probe of brain function in people with prolonged disorders of consciousness, Neuroimage Clin. 12 (2016) 312-319, https://doi.org/10.1016/j.nicl.2016.07.013.

[18] F.M. Noori, N. Naseer, N.K. Qureshi, H. Nazeer, R.A. Khan, Optimal feature selection from fNIRS signals using genetic algorithms for BCI, Neurosci. Lett. 647 (2017) 61-66, https://doi.org/10.1016/j.neulet.2017.03.013.

[19] D. Fernández-Espejo, L. Norton, A.M. Owen, The clinical utility of fMRI for identifying covert awareness in the vegetative state: a comparison of sensitivity between 3T and 1.5T, PLoS One 9 (2014) e95082, , https://doi.org/10.1371/journal.pone. 0095082.

[20] M. Boly, M.R. Coleman, M.H. Davis, A. Hampshire, D. Bor, G. Moonen, P.A. Maquet, J.D. Pickard, S. Laureys, A.M. Owen, When thoughts become action: an fMRI paradigm to study volitional brain activity in non-communicative brain injured patients, Neuroimage 36 (2007) 979-992, https://doi.org/10.1016/j.neuroimage. 2007.02.047.

[21] M.M. Monti, A. Vanhaudenhuyse, M.R. Coleman, M. Boly, J.D. Pickard, L. Tshibanda, A.M. Owen, S. Laureys, Willful modulation of brain activity in disorders of consciousness, N. Engl. J. Med. 362 (2010) 579-589, https://doi.org/10 1056/NEJMoa0905370.

[22] A. Abdalmalak, D. Milej, M. Diop, M. Shokouhi, L. Naci, A.M. Owen, K.St. Lawrence, Can time-resolved NIRS provide the sensitivity to detect brain activity during motor imagery consistently? Biomed. Opt. Express 8 (2017) 2162, https://doi.org/10.1364/BOE.8.002162.

[23] D. Milej, A. Abdalmalak, L. Desjardins, H. Ahmed, T.-Y. Lee, M. Diop, K.St. Lawrence, Quantification of blood-brain barrier permeability by dynamic contrast-enhanced NIRS, Sci. Rep. 7 (2017) 1702, https://doi.org/10.1038/s41598017-01922-x.

[24] D. Milej, A. Abdalmalak, P. McLachlan, M. Diop, A. Liebert, K.St. Lawrence, Subtraction-based approach for enhancing the depth sensitivity of time-resolved NIRS, Biomed. Opt. Express 7 (2016) 4514, https://doi.org/10.1364/BOE.7. 004514.

[25] A. Abdalmalak, D. Milej, M. Diop, L. Naci, A.M. Owen, K.St. Lawrence, Assessing the feasibility of time-resolved fNIRS to detect brain activity during motor imagery, Proc. SPIE. Int. Soc. Opt. Eng. (2016) 969002, , https://doi.org/10.1117/12. 2209587.

[26] A. Liebert, H. Wabnitz, D. Grosenick, M. Möller, R. Macdonald, H. Rinneberg, Evaluation of optical properties of highly scattering media by moments of distributions of times of flight of photons, Appl. Opt. 42 (2003) 5785-5792, https:// doi.org/10.1364/AO.42.005785.

[27] A. Liebert, H. Wabnitz, J. Steinbrink, H. Obrig, M. Möller, R. Macdonald, A. Villringer, H. Rinneberg, Time-resolved multidistance near-infrared spectroscopy of the adult head: intracerebral and extracerebral absorption changes from moments of distribution of times of flight of photons, Appl. Opt. 43 (2004) 3037-3047, https://doi.org/10.1364/AO.43.003037.

[28] F. Scholkmann, S. Spichtig, T. Muehlemann, M. Wolf, How to detect and reduce movement artifacts in near-infrared imaging using moving standard deviation and spline interpolation, Physiol. Meas. 31 (2010) 649-662, https://doi.org/10.1088/ 0967-3334/31/5/004.

[29] Q. Fang, Mesh-based Monte Carlo method using fast ray-tracing in Plücker coordinates, Biomed. Opt. Express 1 (2010) 165, https://doi.org/10.1364/BOE.1. 000165.

[30] V. Fonov, A. Evans, R. McKinstry, C. Almli, D. Collins, Unbiased nonlinear average age-appropriate brain templates from birth to adulthood, Neuroimage. 47 (2009) S102, https://doi.org/10.1016/S1053-8119(09)70884-5.

[31] M. Jäger, A. Kienle, Non-invasive determination of the optical properties of the human head using a neural network, Diffus. Opt. Imaging III (2011) $80880 \mathrm{H}$, https://doi.org/10.1364/ECBO.2011.80880H OSA, Washington, D.C.

[32] A. Shmuel, E. Yacoub, J. Pfeuffer, P.F. Van de Moortele, G. Adriany, X. Hu, K. Ugurbil, Sustained negative BOLD, blood flow and oxygen consumption response and its coupling to the positive response in the human brain, Neuron. $36(2002)$ 1195-1210, https://doi.org/10.1016/S0896-6273(02)01061-9.

[33] M. Čeko, J.L. Gracely, M.-A. Fitzcharles, D.A. Seminowicz, P. Schweinhardt, M.C. Bushnell, Is a responsive default mode network required for successful working memory task performance? J. Neurosci. 35 (2015) 11595-11605, https:// doi.org/10.1523/JNEUROSCI.0264-15.2015.

[34] R.J. Cooper, M. Caffini, J. Dubb, Q. Fang, A. Custo, D. Tsuzuki, B. Fischl, W. Wells, I. Dan, D.A. Boas, Validating atlas-guided DOT: a comparison of diffuse optical tomography informed by atlas and subject-specific anatomies, Neuroimage. 62 (2012) 1999-2006, https://doi.org/10.1016/j.neuroimage.2012.05.031.

[35] X. Xiao, H. Zhu, W.-J. Liu, X.-T. Yu, L. Duan, Z. Li, C.-Z. Zhu, Semi-automatic 10/20 identification method for MRI-Free probe placement in transcranial brain mapping techniques, Front. Neurosci. 11 (2017) 4, https://doi.org/10.3389/fnins.2017. 00004.

[36] T.-S. Noh, Y.-C. Rah, J.S. Kyong, J.S. Kim, M.K. Park, J.H. Lee, S.H. Oh, C.K. Chung, M.-W. Suh, Comparison of treatment outcomes between 10 and 20 EEG electrode location system-guided and neuronavigation-guided repetitive transcranial magnetic stimulation in chronic tinnitus patients and target localization in the Asian brain, Acta Otolaryngol. 137 (2017) 945-951, https://doi.org/10.1080/00016489. 2017.1316870.

[37] H. Dehghani, B.R. White, B.W. Zeff, A. Tizzard, J.P. Culver, Depth sensitivity and image reconstruction analysis of dense imaging arrays for mapping brain function with diffuse optical tomography, Appl. Opt. 48 (2009) D137-43 http://www.ncbi. nlm.nih.gov/entrez/query.fcgi?cmd = Retrieve\%7B\&\%7Ddb = PubMed\%7B\& \%7Ddopt $=$ Citation\%7B\&\%7Dlist\%7B_\%7Duids = 19340101 .

[38] H.-D. Nguyen, K.-S. Hong, Bundled-optode implementation for 3D imaging in functional near-infrared spectroscopy, Biomed. Opt. Express 7 (2016) 3491, https://doi.org/10.1364/BOE.7.003491.

[39] S.H. Jang, J.P. Seo, S.H. Ahn, M.Y. Lee, Comparison of cortical activation patterns by somatosensory stimulation on the palm and dorsum of the hand, Somatosens. Mot. Res. (2013), https://doi.org/10.3109/08990220.2013.775117. 